\title{
Microarray analysis reveals long non-coding RNA SOX2OT as a novel candidate regulator in diabetic nephropathy
}

\author{
XIAOXUE ZHANG $^{1 *}$, JIN SHANG ${ }^{1 *}$, XIAOYANG WANG $^{1}$, GENYANG CHENG $^{1}$, \\ YUMIN JIANG $^{2}$, DONG LIU ${ }^{1}$, JING XIAO $^{1}$ and ZHANZHENG ZHAO ${ }^{1}$ \\ Departments of ${ }^{1}$ Nephrology and ${ }^{2}$ Emergency, The First Affiliated Hospital of \\ Zhengzhou University, Zhengzhou, Henan 450052, P.R. China
}

Received April 20, 2018; Accepted September 14, 2018

DOI: $10.3892 / \mathrm{mmr} .2018 .9534$

\begin{abstract}
Diabetic nephropathy (DN) is a highly complex syndrome involving multiple dysregulated biological processes. Long non-coding RNAs (lncRNAs) are now believed to have an important function in various diseases. However, their roles in DN remain largely unknown. Therefore, the present study was performed in order to investigate the lncRNAs that have a crucial role in DN. $\mathrm{db} / \mathrm{db}$ mice were used as a DN model while $\mathrm{db} / \mathrm{m}$ mice served as a control to search for lncRNAs which may have important roles in DN. Microarray and bioinformatics analysis gave an overview of the features of differentially expressed genes. Gene Ontology and Kyoto Encyclopedia of Genes and Genomes enrichment analysis demonstrated the typical biological alterations in DN. A co-expression network of IncRNAs and mRNAs revealed the complex interaction pattern in DN conditions. Further data investigation indicated that SOX2-overlapping transcript (SOX2OT), which was significantly downregulated in DN mice, may be the potentially functional lncRNA contributing to the onset of DN. The UCSC database demonstrated that SOX2OT was highly conserved in mice and humans. Additionally further study using cultured human podocytes and mesangial cells confirmed the downregulation of SOX2OT using reverse transcription-quantitative polymerase chain reaction and fluorescence in situ hybridization. However, the cellular location of SOX2OT depended on certain cell types. Taken together, the results of the present study indicated that SOX2OT may act as an important regulator in the pathogenesis of DN by interacting with various mRNAs with critical roles in DN.
\end{abstract}

Correspondence to: Professor Zhanzheng Zhao, Department of Nephrology, The First Affiliated Hospital of Zhengzhou University, 1 of East Jianshe Road, Zhengzhou, Henan 450052, P.R. China E-mail: zhanzhengzhao@zzu.edu.cn

\section{*Contributed equally}

Key words: SOX2-overlapping transcript, diabetic nephropathy, microarray analysis, bioinformatics analysis

\section{Introduction}

Diabetic nephropathy (DN), as a major microvascular complication of diabetes, has become the leading cause of renal decompensation and failure worldwide $(1,2)$. The global prevalence of type 2 diabetes contributes to an increasing number of patients developing DN and eventually end-stage renal disease, which requires renal replacement therapy. There are a number of physiological and molecular alterations in the renal microenvironment during DN, including oxidative stress, activation of certain signaling pathways, inflammation and increasing matrix accumulation (3-5). However, the underlying mechanism leading to these changes has not been fully identified. Further understanding of the molecular mechanisms involved in DN is of clinical importance in order to improve the therapeutic strategies for DN.

Long noncoding RNAs (lncRNAs) are a class of transcripts longer than 200 nucleotides without protein coding function. The mammalian genomes are replete with lncRNA genes that are engaged in numerous biological processes (6). LncRNAs differ from protein-coding genes in a number of ways. In particular, they are frequently not as conserved compared with mRNAs at the level of the primary sequence and the function of most lncRNAs remains unknown, which is why they are dismissed to be the 'dark matter' of the transcriptome. Recent studies have identified the regulatory role of lncRNA in a number of gene expression processes, including nuclear importation, alternative splicing, DNA methylation, mRNA decay, as well as other transcriptional, post-transcriptional and epigenetic regulatory roles $(7,8)$. Although a number of lncRNA molecules have been reported to serve crucial roles in diverse diseases, only limited examples of lncRNAs have been described in DN.

In the present study, the differentially expressed genes (DEGs) were initially screened for lncRNAs and mRNAs, between $\mathrm{db} / \mathrm{db}$ mice and $\mathrm{db} / \mathrm{m}$ mice by microarray technique. Bioinformatics analysis identified several lncRNAs that putatively served an important role in the regulation of mRNAs involved in DN onset of db/db mice. By searching associated databases and analyzing the results SOX2OT, which has never been reported in DN was selected and focused on. SOX2OT was downregulated and highly conserved between mice and humans. Gene-lncRNA co-expression networks were 
constructed to investigate the putative function and interaction patterns of SOX2OT. To study the role of SOX2OT in DN, human podocyte and mesangial cells were also cultured to determine SOX2OT expression pattern under high glucose conditions, aiming at providing novel view on DN pathogenesis and identifying novel directions for further research.

\section{Materials and methods}

Animal studies. C57BLKS/J background Lepr ${ }^{\mathrm{db}} / \operatorname{Lepr}^{\mathrm{db}}(\mathrm{db} / \mathrm{db}$; $\mathrm{n}=3)$ and the non-diabetic control Lepr $\mathrm{db} / \mathrm{m}(\mathrm{db} / \mathrm{m} ; \mathrm{n}=3)$ male mice (aged 8 weeks old; weight, 38-40 g) were bought from the Model Animal Research Center of Nanjing University (Nanjing, China). Mice were housed at $20-25^{\circ} \mathrm{C}$, a humidity of $30-60 \%$ and a $12 / 12 \mathrm{~h}$ light/dark cycle. $\mathrm{db} / \mathrm{db}$ is a spontaneous diabetic model with uncontrolled high blood glucose. All rats had unrestricted access to food/water and were maintained for 20 weeks. Body weight, fasting blood glucose, urinary protein/creatinine ratio, serum creatinine and blood urea nitrogen were repeatedly measured to monitor the onset and progression of DN. At the termination of the study, mice were sacrificed under deep anesthesia prior to collecting blood (0.5-1 ml) and tissue samples. Fasting blood glucose was measured by quick sticks using tail blood. Urinary protein, serum creatinine and blood urea nitrogen were measured by the clinical laboratory of the First Affiliated Hospital of Zhengzhou University. All protocols were approved by the Institutional Animal Care and Use Committee of the First Affiliated Hospital of Zhengzhou University and conducted in accordance with the National Institutes of Health (NIH) Guide for the Care and Use of Laboratory Animals.

Cell culture and treatment. Human podocytes cells (HPCs) and human mesangial cells (HMCs) were a gift from Professor Fan Yi, Shandong University (Jinan, China). HPCs and HMCs were cultured as previously described $(9,10)$. All cells were cultured in the Dulbecco's modified Eagle's medium supplied with $10 \%$ fetal bovine serum (both Gibco; Thermo Fisher Scientific, Inc., MA, USA) at $37^{\circ} \mathrm{C}$ with $5 \% \mathrm{CO}_{2}$. High glucose (HG, final concentration $30 \mathrm{mM}$ in culture medium) was used in the present study and the same concentration of mannitol was used as an osmolality control.

Hematoxylin-eosin and periodic Acid-Schiff staining. Tissue samples of renal cortex were fixed in buffered formaldehyde solution for $24 \mathrm{~h}$ at room temperature, dehydrated and embedded in paraffin wax. Sections were cut $(4 \mu \mathrm{M})$ and stained with hematoxylin-eosin (HE; OriGene Technologies, Inc., Beijing, China) and Periodic Acid-Shiffs (PAS, Solarbio Science and Technology Co., Ltd., Beijing, China) reagent. In HE staining, samples were treated with hematoxylin for $8 \mathrm{~min}$ and $1 \%$ ethanol eosin for another $3 \mathrm{~min}$. In PAS staining, slices were oxidized by $0.5 \%$ periodic acid for $7 \mathrm{~min}$ and soak in $0.5 \%$ sodium metabisulfite for $1 \mathrm{~min}$. Sections were mounted by neutral balsam $(11,12)$. Images were captured by Leica Microsystems GmbH, (Wetzlar, Germany).

Transmission electron microscopy observation. Ultramicrostructure of the basement membrane region was observed by transmission electron microscopy (TEM) (13). Tissues for TEM observation were fixed in
$2 \%$ glutaraldehyde $/ 2 \%$ paraformaldehyde in $0.1 \mathrm{~mol} / 1$ phosphate buffer for $>24 \mathrm{~h}$ at room temperature, post-fixed in buffered osmic acid, dehydrated in graded alcohols and embedded in an Epon 812 mixture overnight at room temperature. Semi-thin sections $(2 \mu \mathrm{m})$ were rinsed overnight in $0.1 \mathrm{M}$ phosphate buffer, post-fixed for $2 \mathrm{~h}$ in $1 \%$ osmium tetroxide at room temperature, dehydrated and then embedded in Araldite mixture. Ultrathin sections were stained with uranyl acetate at room temperature for $15 \mathrm{~min}$ and lead citrate at room temperature for $25 \mathrm{~min}$, and then examined with a CM10 transmission electron microscope (Philips Medical Systems B.V., Eindhoven, The Netherlands).

Microarray analysis. Agilent SurePrint G3 Mouse Gene Expression v2 $8 \times 60 \mathrm{~K}$ kit, which could scan the signals of 27122 Entrez Gene RNAs and 4578 lncRNAs, was used in the present study. Total RNA of the renal cortex of the mice was extracted and purified using mirVana ${ }^{\mathrm{TM}}$ miRNA Isolation kit (cat. no. AM1561; Ambion; Thermo Fisher Scientific, Inc.) and checked for a RNA integrity Number (RIN) to inspect RNA integration by an Agilent Bioanalyzer 2100 (Agilent Technologies, Inc., Santa Clara, CA, USA). Total RNA was amplified and labeled by Low Input Quick Amp Labeling kit, One-Color (cat. no. 5190-2305; Agilent Technologies, Inc.). Labeled cRNA were purified using an RNeasy mini kit (cat. no. 74106; Qiagen, GmBH, Germany). Each slide was hybridized with 600 ng Cy3-labeled cRNA using Gene Expression Hybridization kit (cat. no. 5188-5242; Agilent Technologies, Inc.). Following $17 \mathrm{~h}$ hybridization, slides were washed in staining dishes (cat. no. 121; Thermo Fisher Scientific, Inc.) with Gene Expression Wash Buffer kit (cat. no. 5188-5327, Agilent Technologies, Inc.). Slides were scanned by Agilent Microarray Scanner (cat. no. G2565CA; Agilent Technologies, Inc.) with default settings. Data were extracted with Feature Extraction software 10.7 (Agilent Technologies, Inc.). All experiments were performed according to the manufacturer's protocols (14).

Reverse transcription-quantitative polymerase chain reaction (RT-qPCR) analysis. Total RNA of the renal cortices of the mice was extracted using TRIzol solution (Takara Biotechnology Co., Ltd., Dalian, China). Then total RNA was quantified using a Nanodrop 2000 spectrophotometer (Thermo Fisher Scientific, Inc.). A total of $1 \mu \mathrm{g}$ of RNA was used for cDNA synthesis using PrimeScript RT Master Mix (Perfect Real Time) kit (Takara Biotechnology Co., Ltd.). The temperature protocol for RT was $37^{\circ} \mathrm{C}$ for $15 \mathrm{~min}$ followed by $85^{\circ} \mathrm{C}$ for $5 \mathrm{sec}$. SYBR Premix Ex Taq kit (Takara Biotechnology Co., Ltd.) was used to evaluate the lncRNA and mRNA expression (15). RT-qPCR was performed in a StepOnePlus Real-Time PCR System (Applied Biosystems; Thermo Fisher Scientific, Inc.). The specific primers of target genes used were as follows: Mus Klk1b7-ps forward, 5'-GTG TGAACCTCAAGCTCCTG-3' and reverse, 5'-CTGAGT CTCCCTTGCAAGTG-3'; Mus Suv39h2 forward, 5'-TGT GTGCCTTGCCTAGTTTC-3' and reverse, 5'-CCACACCCT TTGCTACCTTG-3'; Mus Ubtf forward, 5'-TGCGGTTCC TTGAGAGCTTG-3' and reverse, 5'-CACCCTTGCCACCTT CCTG-3'; Mus Pkib forward, 5'-TGGCCGTGAAGGAAG ATGC-3' and reverse, 5'-ACTACCAGATCAAACACCCC-3'; Mus 1700112J16Rik forward, 5'-TGACCCAGATATCAG TGCTC-3' and reverse, 5'-TGTCTCTTCCTGTTCTGG TC-3'; Mus Gm15217 forward, 5'-TGGATCACAAGGACC 
Table I. Data of mice at the end point of the experiment.

\begin{tabular}{|c|c|c|c|}
\hline Parameter & Control $(n=3)$ & $\mathrm{DN}(\mathrm{n}=3)$ & P-value \\
\hline Body weight (g) & $23.27 \pm 0.30$ & $39.20 \pm 0.35$ & $<0.0001$ \\
\hline Fasting blood glucose (mmol/l) & $7.367 \pm 0.26$ & $30.33 \pm 1.44$ & $<0.0001$ \\
\hline Urinary protein/creatinine ratio $(\mathrm{g} / \mathrm{g})$ & $1.477 \pm 0.12$ & $13.32 \pm 1.80$ & 0.0028 \\
\hline Serum creatinine $(\mu \mathrm{mol} / \mathrm{l})$ & $33.30 \pm 3.30$ & $41.47 \pm 1.38$ & 0.0842 \\
\hline Blood urea nitrogen (mmol/l) & $8.627 \pm 0.47$ & $13.38 \pm 0.25$ & 0.0009 \\
\hline
\end{tabular}

Control, nondiabetic $\mathrm{db} / \mathrm{m}$ mice; $\mathrm{DN}$, diabetic $\mathrm{db} / \mathrm{db}$ mice. $\mathrm{P}<0.05$ is considered as statistically different between two groups. Values are presented as the mean \pm standard deviation.

CCAGT-3' and reverse, 5'-GAACCAAATCCAACGGAG AC-3'; Mus Pvt1 forward, 5'-AGAGCTGGTAGGAGACAG AC-3' and reverse, 5'-TCTGCCAGCTCCTTCTTCAC-3'; Mus Hbegf forward, 5'-CTCCCTCTTGCAAATGCCTC-3' and reverse, 5'-AGTACTACAGCCACCACAGC-3'; Mus Hsd11b1 forward, 5'-GAGTTCAGACCAGAAATGCTC-3' and reverse, 5'-AGACACTACCTTCTGGAGAC-3'; Mus Sox 2ot forward, 5'-CCGAGA AGCAAACCTGACAG-3' and reverse, 5'-AGTCTCTCCCATCAGCGTC-3'; Homo LncR-SOX2-OT forward, 5'-AGACAATGAGCTGGCACC AC-3' and reverse, 5'-AGGCAAGGTCAGAGACATAG-3'. Housekeeping gene GAPDH was used as an endogenous control for normalization of RNA quantity differences. The cycling conditions were as follows: $95^{\circ} \mathrm{C}$ for $30 \mathrm{sec}, 40$ cycles at $95^{\circ} \mathrm{C}$ for $5 \mathrm{sec}$ and then $60^{\circ} \mathrm{C}$ for $30 \mathrm{sec}$. All reactions were carried out in triplicate. Relative expression of gene levels were quantified using the $2^{-\Delta \Delta \mathrm{Cq}}$ method (16).

Gene ontology (GO) and pathway enrichment analysis. GO enrichment analysis was performed to categorize the differentially expressed mRNAs based on biological processes, molecular functions and cellular components. The Kyoto Encyclopedia of Genes and Genomes (KEGG) database was used to investigate the enriched pathways of aberrantly expressed mRNAs $(17,18)$. $\mathrm{P}<0.05$ was considered to indicate a statistically significant difference.

Co-expression of lncRNAs and mRNAs. Currently most IncRNAs have not been functionally annotated. Therefore, their functions were predicted on the basis of the functional annotations of their co-expressed mRNAs. Pearson coefficients (PC) were used to construct a network of 1 ncRNAs and mRNAs. Pair-wise PC of genes (e.g., lncRNA and lncRNA, lncRNA and mRNA, mRNA and mRNA) were used to construct networks using Cytoscape 3.5.1 (www.cytoscape.org) using the following steps: i) Choose interactions of IncRNA and mRNA to construct initial networks $(\mathrm{P}<0.001)$, genes presented in network were considered as 'existed'. ii) Simultaneously import interactions of existed 'IncRNA and lncRNA', existed 'mRNA and mRNA' into initial networks $(\mathrm{P}<0.001)$.

Fluorescence in situ hybridization (FISH). To detect lncRNA SOX2OT expression pattern of HPC and HMC under normal or HG-stimulation conditions, FISH was performed as previously described (19). The probe and kit for FISH was purchased from
Guangzhou RiboBio Co., Ltd., (Guangzhou, China). Cultured HPCs and HMCs were fixed in paraformaldehyde for $10 \mathrm{~min}$ at room temperature and incubated in Triton X-100 for 5 min. Non-specific binding sites were blocked using pre-hybridization buffer for $30 \mathrm{~min}$ at $37^{\circ} \mathrm{C}$. A biotin-labeled SOX2OT probe was used at $5 \mathrm{nM}$. Images were observed and captured using a fluorescence microscope (Leica Microsystems $\mathrm{GmbH}$ ).

Statistical analysis. Data are reported as the mean \pm standard deviation. Experiments were performed in triplicate. One-way analysis of variance followed by Duncan's multiple range test were used to check the significance of the differences in mean values. Statistical analyses were performed with GraphPad Prism 5.0 (La Jolla, CA, USA). P $<0.05$ was considered to indicate a statistically significant difference in microarray analysis and if fold-changes were $>2$ or $<0.5, \mathrm{P}$ values were corrected by the Benjamini-Hochberg false discovery rate method using R software (version 3.5.1; https://www.r-project.org/).

\section{Results}

Metabolic and pathological characteristics of $\mathrm{db} / \mathrm{db}$ mice. Table I described detailed laboratory alterations of the mice at the end of the study. The $\mathrm{db} / \mathrm{db}$ mice demonstrated higher body weight, fasting blood glucose and urinary protein level compared with the $\mathrm{db} / \mathrm{m}$ group $(\mathrm{P}<0.05)$. Serum creatinine level and blood urea nitrogen also increased compared with the $\mathrm{db} / \mathrm{m}$ mice. However, the serum creatinine level was not statistically significant. HE staining, PAS staining and TEM (Fig. 1A) were used for morphological diagnosis of DN. $\mathrm{Db} / \mathrm{db}$ mice exhibited foot process enfacement of podocytes, thickening of the basement membrane and expansion of the mesangial matrix. All these results indicated that a successful $\mathrm{DN}$ model of $\mathrm{db} / \mathrm{db}$ mice had been achieved.

Microarray analysis reveals the DEGs of $\mathrm{db} / \mathrm{db}$ mice compared with $\mathrm{db} / \mathrm{m}$ mice. The microarray gene chip data demonstrated 11,673 DEGs in $\mathrm{db} / \mathrm{db}$ mice compared with $\mathrm{db} / \mathrm{m}$ mice. A total of 5,233 genes were upregulated while 6,440 were downregulated. Further study demonstrated 1,568 DEGs with fold-change $>2.0$. Among those, 777 were consistently upregulated and 791 were consistently downregulated. A hierarchically clustered heat map is presented in Fig. 1B.Sec1413 (NM_001029937) and Gm6300 (NR_033591) were the most downregulated mRNA and lncRNA, with 
A
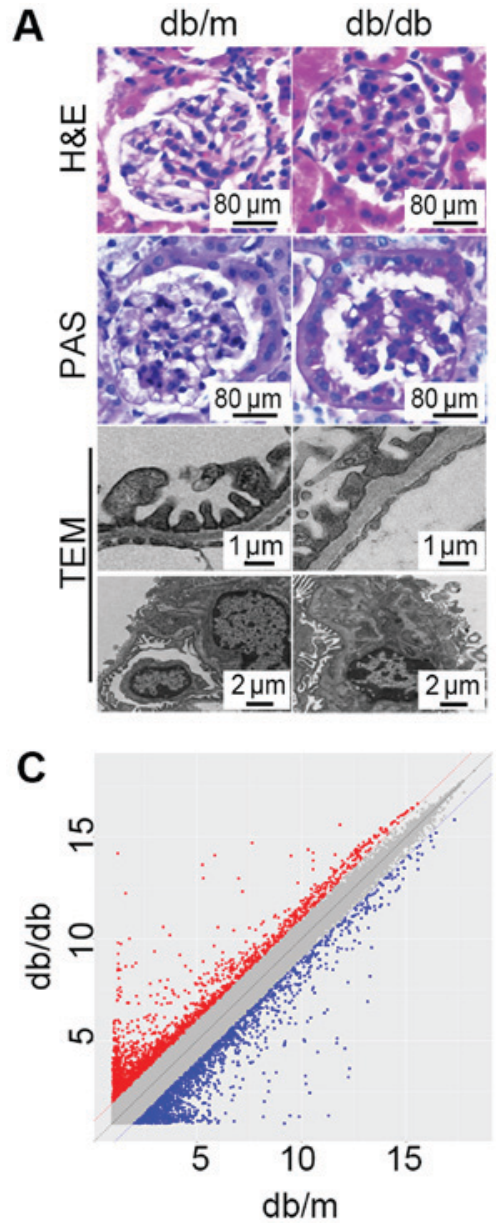

B

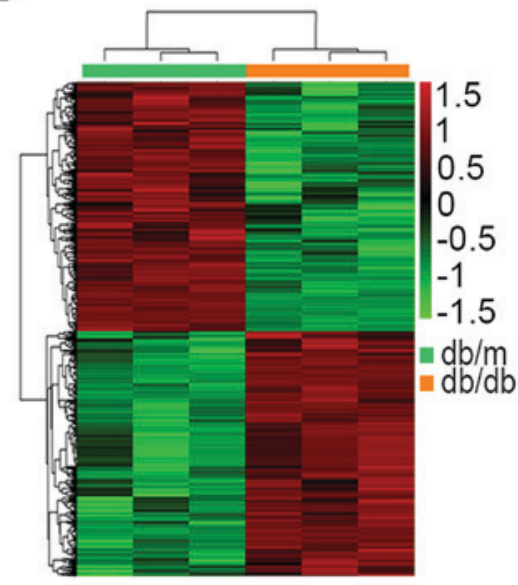

D

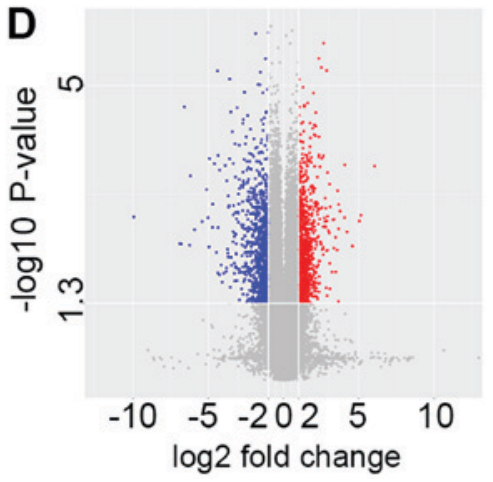

Figure 1. Microarray analysis revealed DEGs between $\mathrm{db} / \mathrm{db}$ mice and $\mathrm{db} / \mathrm{m}$ mice. (A) Representative morphological alterations, including H\&E staining, PAS base staining and TEM inspection, in the renal cortex from $\mathrm{db} / \mathrm{m}(\mathrm{n}=3)$ and $\mathrm{db} / \mathrm{db}(\mathrm{n}=3)$ mice. Scale bars are presented in each graph. (B) Hierarchically clustered heat map of DEGs with absolute fold-changes of no less than 2.0. The vertical axis represents each mRNA or long noncoding RNA and the horizontal axis represents different groups. (C) A scatter plot exhibiting the DEGs between $\mathrm{db} / \mathrm{db}$ mice and db/m mice. (D) A volcano plot exhibiting statistically significant DEGs (fold-change $\geq 2.0, \mathrm{P}<0.05$ ). DEGs, differentially expressed genes; PAS, periodic Acid Schiff; H\&E, hematoxylin and eosin; TEM, transmission electron microscopy; db/db, Lepr ${ }^{\mathrm{db}} / \mathrm{Lepr}^{\mathrm{db}}$.

an absolute fold-change of 118.84 and 59.97, respectively. Hmgcs2 (NM_008256) and Rdh18-ps (NR_037604) were the most upregulated mRNA and lncRNA with fold-changes of 23.81and 5.13. Detailed DEGs of mRNAs and lncRNAs with the greatest change are listed in Table II. The scatter plot (Fig. 1C) presents variations in DEGs expression between the two groups. Significant expression differences can be seen. The volcano plot (Fig. 1D) was presented to visualize the DEGs with statistical significance (fold-change $\geq 2.0, \mathrm{P}<0.05$ ).

GO and pathway enrichment analysis of differently expressed mRNAs. GO analysis (Fig. 2A) was performed in order to investigate the important biological processes, cellular components and molecular functions involved in the onset of DN. The most enriched biological process was the glutathione biosynthetic process. Glucuronic and flavonoid associated processes were also enriched, including glucoronate metabolic processes, cellular glucuronidation, flavonoid metabolic processes, flavonoid glucuronidation and flavonoid biosynthetic processes. Chylomicron was the most enriched cellular component. The most enriched molecular functions were inorganic anion exchanger activity and sodium-independent organic anion transmembrane transporter activity.
The top 30 enriched pathways are presented in Fig. 2B. The most enriched pathway was biotin metabolism. However, it only contained one DEG (OXSM). Cytochrome P450-associated pathways were enriched, including metabolism of xenobiotics by cytochrome P450 and drug metabolism-cytochrome P450. Certain well-known pathways that are associated with the onset and progression of DN could also been observed, including the renin-angiotensin system, PPAR signaling pathway, glutathione metabolism biosynthesis of unsaturated fatty acids and arachidonic acid metabolism.

It is notable that in GO and pathway enrichment analysis demonstrated alterations in steroid associated enrichment, suggesting that steroid hormone disorder may also participate in the onset of DN.

Differentially expressed IncRNA and mRNA have close associations. Of all these DEGs, 880 mRNAs (335 were upregulated and 545 were downregulated) and 60 lncRNAs (30 were upregulated and 30 were downregulated) were annotated. Hierarchically clustered heat maps are presented in Fig. 3A and B. To predict the function of differential lncRNAs, PCs of each pair of differentially expressed lncRNAs and mRNAs were calculated for the first step. The association of 
Table II. Top 10 down and upregulated mRNAs and lncRNAs ( $\mathrm{db} / \mathrm{db}$ compared with $\mathrm{db} / \mathrm{m}$. Only genes with Accession number started with 'NM' or 'NR' are listed here).

\section{mRNAs}

Gene symbol

Sec1413

Slcola1

Slc22a7

Masp2

Cyp7b1

Col19a1

Mfsd2a

Gm853

Alox 15

Akr1c18

Bhmt

Qrfpr

Chrdl2

Grem1

Kynu

Gm10639

Gsta2

Cry1

Cyp4a14

Hmges2

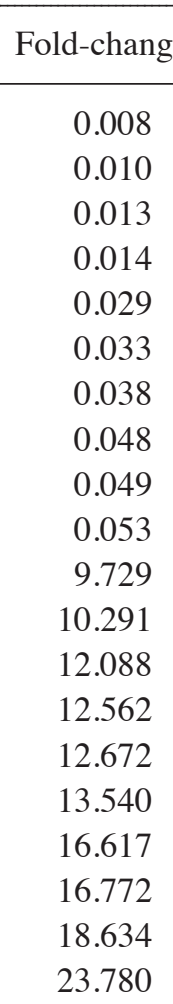

Accession

NM_001029937

NM_013797

NM_144856

NM_010767

NM_007825

NM_007733

NM_029662

NM_001034872

NM_009660

NM_134066

NM_016669

NM_198192

NM_133709

NM_011824

NM_027552

NM_001122660

NM_008182

NM_007771

NM_007822

NM_008256
lncRNAs

\begin{tabular}{lcc}
\hline \multicolumn{1}{c}{ Gene symbol } & Fold-change & Accession \\
Gm6300 & 0.017 & NR_033591 \\
Sox2ot & 0.031 & NR_015580 \\
Gm1653 & 0.042 & NR_040591 \\
0610031O16Rik & 0.080 & NR_045760 \\
Carlr & 0.084 & NR_131254 \\
Gm15350 & 0.157 & NR_045775 \\
B230323A14Rik & 0.172 & NR_040765 \\
Airn & 0.205 & NR_027773 \\
Gm10433 & 0.255 & NR_045282 \\
5830418P13Rik & 0.260 & NR_040466 \\
Gm16796 & 2.884 & NR_040367 \\
1700110I01Rik & 2.920 & NR_038059 \\
2310069G16Rik & 2.954 & NR_040309 \\
Zfp133-ps & 2.966 & NR_033459 \\
A530006G24Rik & 3.089 & NR_046014 \\
A930001A20Rik & 3.101 & NR_040549 \\
I730030J21Rik & 3.270 & NR_045781 \\
BB123696 & 3.466 & NR_027893 \\
K1k1b7-ps & 4.188 & NR_033120 \\
Rdh18-ps & 5.131 & NR_037604 \\
& &
\end{tabular}

lnc, long non-coding.

A

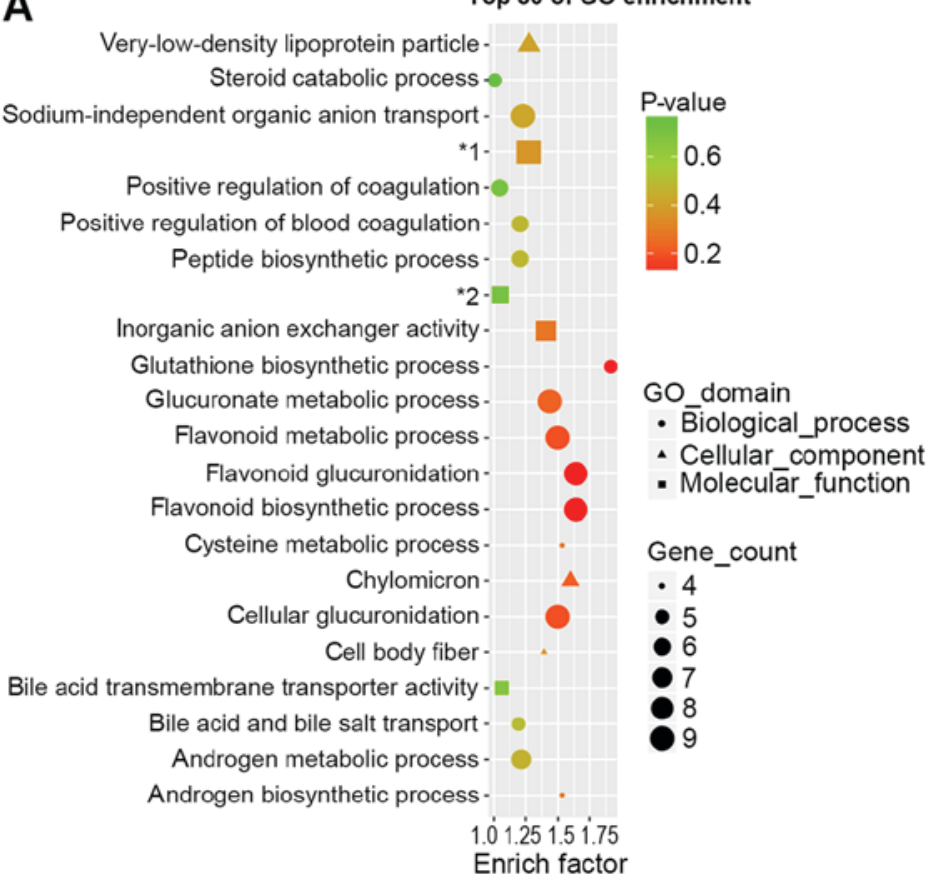

B

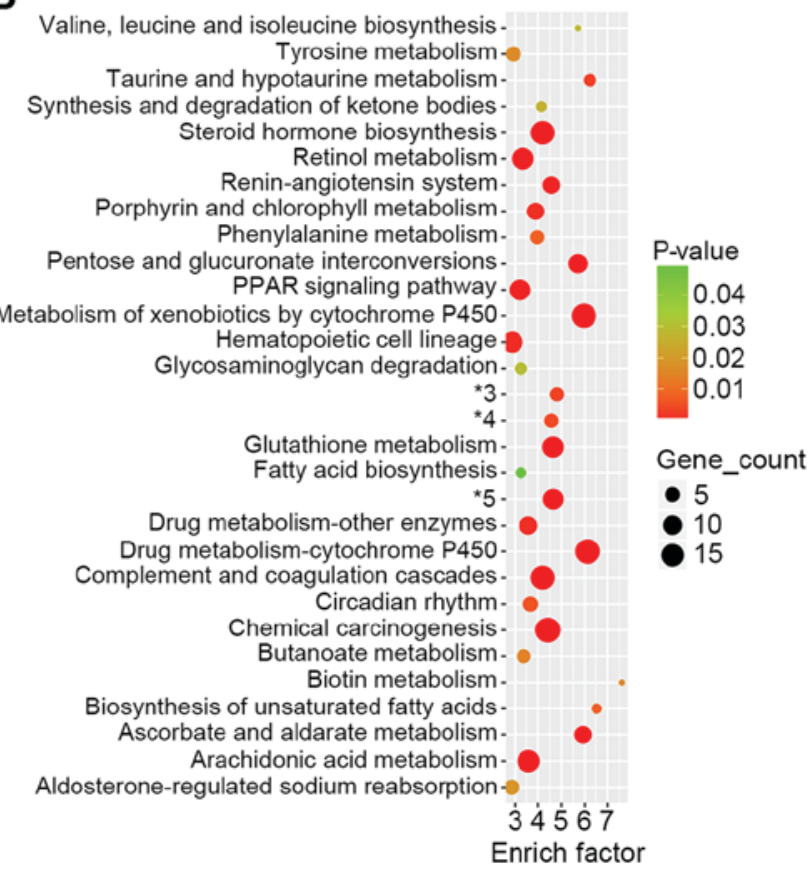

Figure 2. GO and KEGG analysis of differentially expressed mRNAs. (A) Major alterations in mRNA expression were categorized into biological processes, cellular component and molecular function by GO enrichment analysis. (B) KEGG analysis demonstrating the most enriched alterations in complex cellular pathways. *1: Sodium-independent organic anion transport. *2: Oxidoreductase activity, acting on the CH-NH2 group of donors. *3: Glycosaminoglycan biosynthesis-heparan sulfate/heparin. *4: Glycosaminoglycan biosynthesis-chondroitin sulfate/dermatan sulfate. *5: Endocrine and other factor-regulated calcium reabsorption. GO, gene ontology; KEGG, Kyoto encyclopedia of genes and genomes. 
A Color key

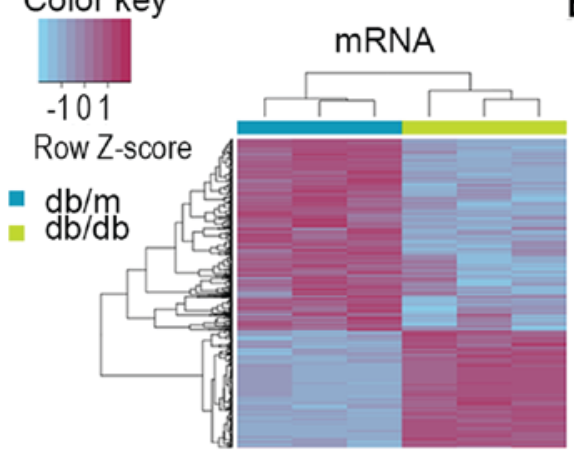

B Color key

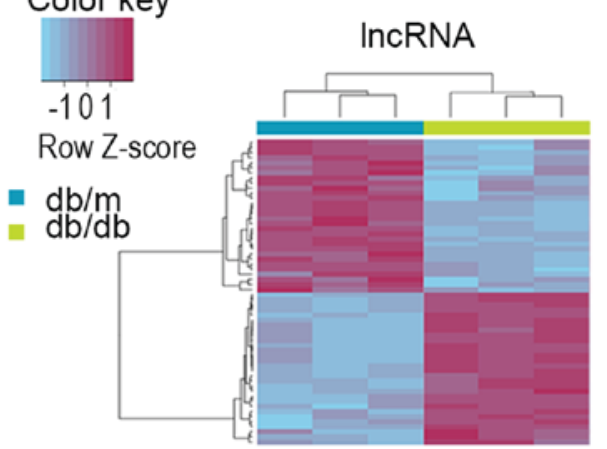

C

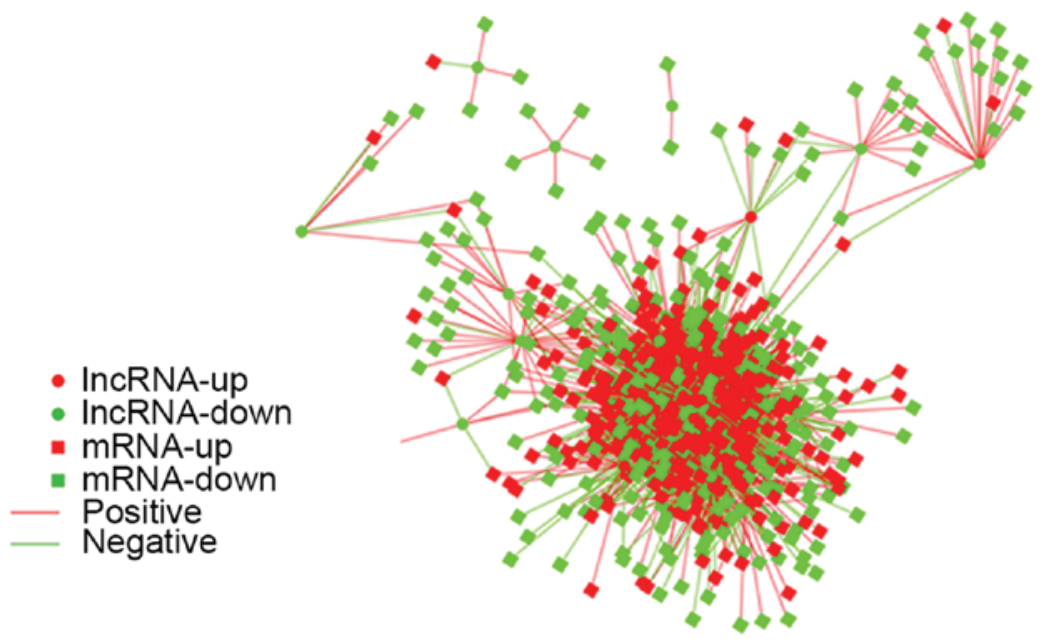

D

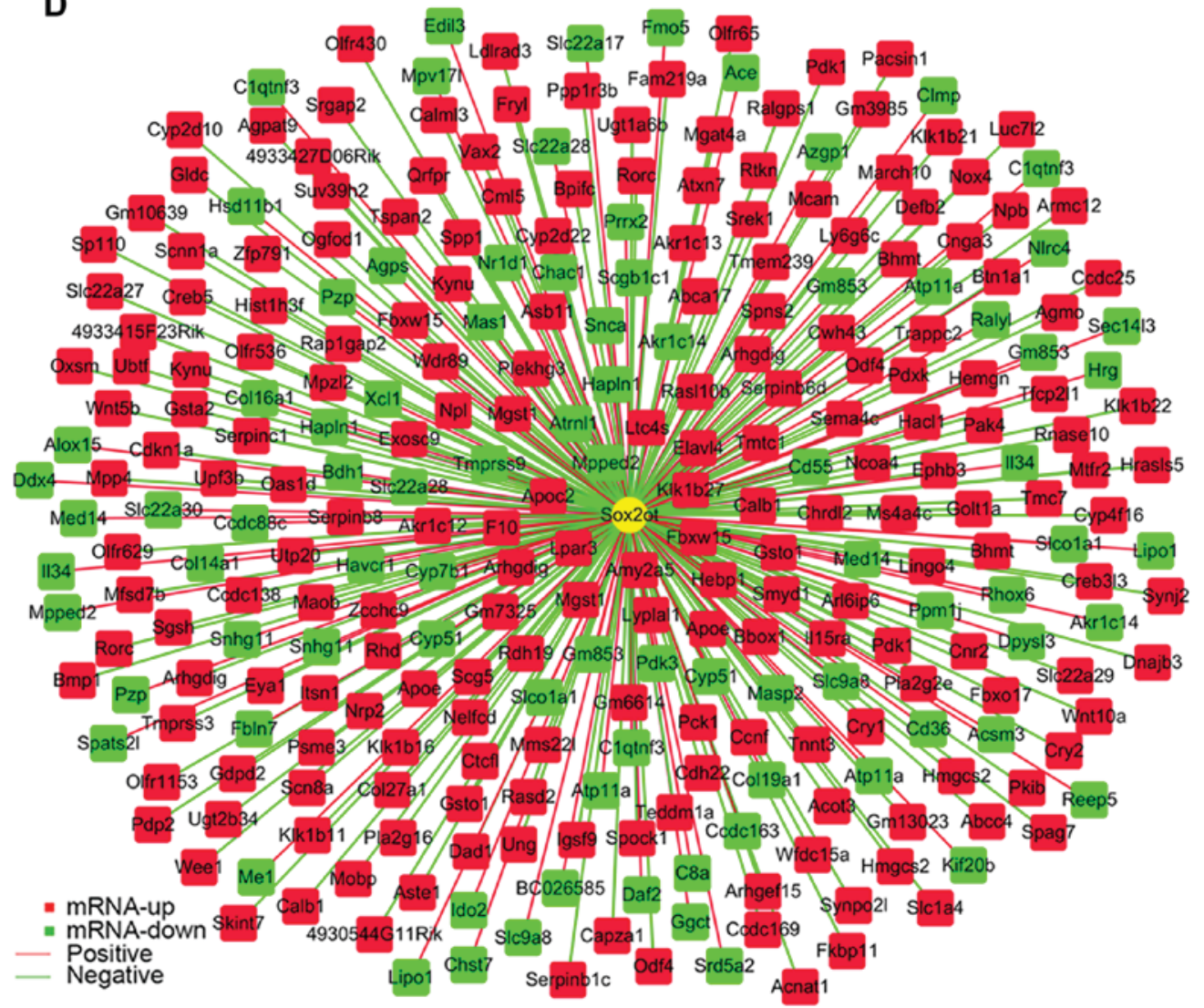

Figure 3. IncRNA and mRNA correlation analysis revealed potential connections between them. Hierarchically clustered heat map of (A) mRNA and (B) lncRNA with fold-changes no less than 2.0 between $\mathrm{db} / \mathrm{db}$ and $\mathrm{db} / \mathrm{m}$ mice. The vertical axis represents each mRNA or lncRNA and the horizontal axis represents different groups. (C) Interaction network of lncRNA and mRNA were constructed using Pearson Coefficients (P $\leq 0.001)$. (D) Interaction network of SOX2OT and its associated genes were constructed using the same method. Circles denote lncRNA and rectangles denote mRNA. Red is enriched in the $\mathrm{db} / \mathrm{db}$ group and green is enriched in the $\mathrm{db} / \mathrm{m}$ group. Red edges denote positive interactions and green edges denote negative interactions. Lnc, long noncoding; SOX2OT, SOX2-original transcript; db/db, Lepr ${ }^{\mathrm{db}} / \mathrm{Lepr}^{\mathrm{db}}$. 
A

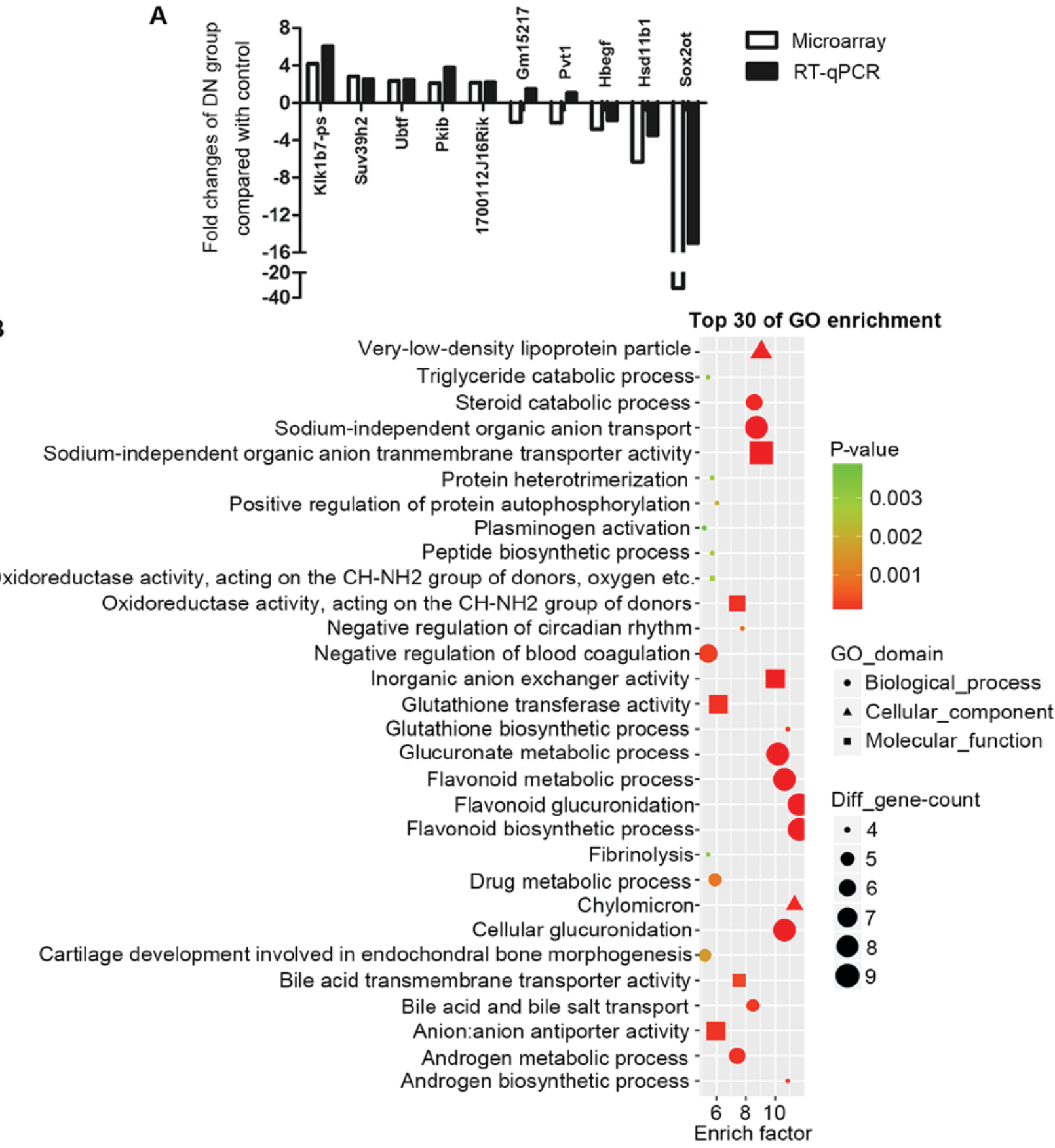

B

Figure 4. Validation of microarray results by RT-qPCR and analysis of SOX2OT function by GO enrichment. (A) Certain mRNAs and highly conserved long noncoding RNAs according to UCSC were chosen for RT-qPCR validation. Results demonstrated consistency between these two methods except for Gm15217 and Pvt1. (B) All the SOX2OT-associated mRNAs were analyzed in GO enrichment. Glutathione metabolic, oxidoreductase activity, flavonoid metabolic and lipid metabolic processes are enriched. RT-qPCR, reverse transcription quantitative polymerase chain reaction; GO, gene ontology; DN, diabetic nephropathy; SOX2OT, SOX2 original transcript.

lncRNAs and mRNA were constructed and intensive correlations $(\mathrm{P} \leq 0.001)$ between them are demonstrated as network in Fig. 3C, indicating lncRNAs may perform their function via interacting with associated mRNAs. To get a better view of the gene network of SOX2OT the IncRNA of interest, a SOX2OT-centered network was constructed (Fig. 3D).

RT-qPCR validation of DEGs. First, the UCSC database was searched to check the homology of all differentially expressed lncRNAs (fold-change>2.0). Highly homologous lncRNAs were chosen for the RT-qPCR validation, including Pvt1, SOX2OT,
Gm15217, Klk1b7-ps and 1700112J16Rik. Certain other randomly picked differentially expressed mRNAs, including: Ubtf, Pkib, Suv39h2, Hsd11b1 and Hbegf, were also included in RT-qPCR validation. Fig. 4A demonstrates that most of the results of RT-qPCR were consistent with microarray results. Out of these 5 lncRNAs, Pvt1 and SOX2OT have already been reported to exist in humans $(20,21)$. Notably, SOX2OT is the most markedly downregulated gene, with a fold-change of -32 .

SOX2OT may have an important role in DN. From Fig. 4A, SOX2OT was identified to be the most dysregulated lncRNA 
A

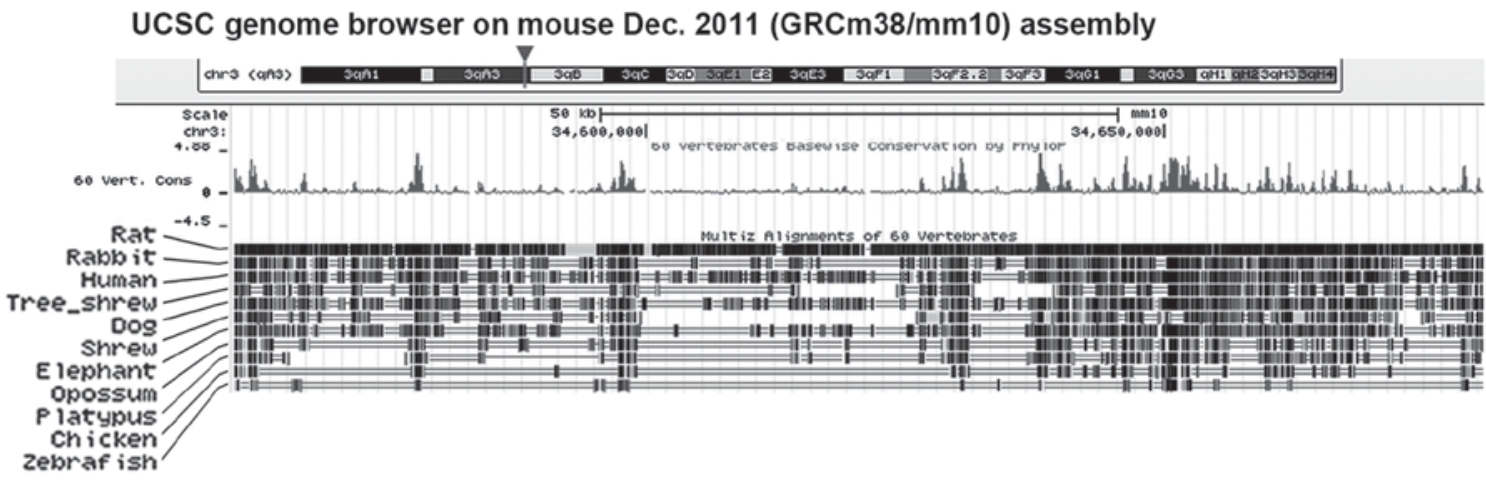

B
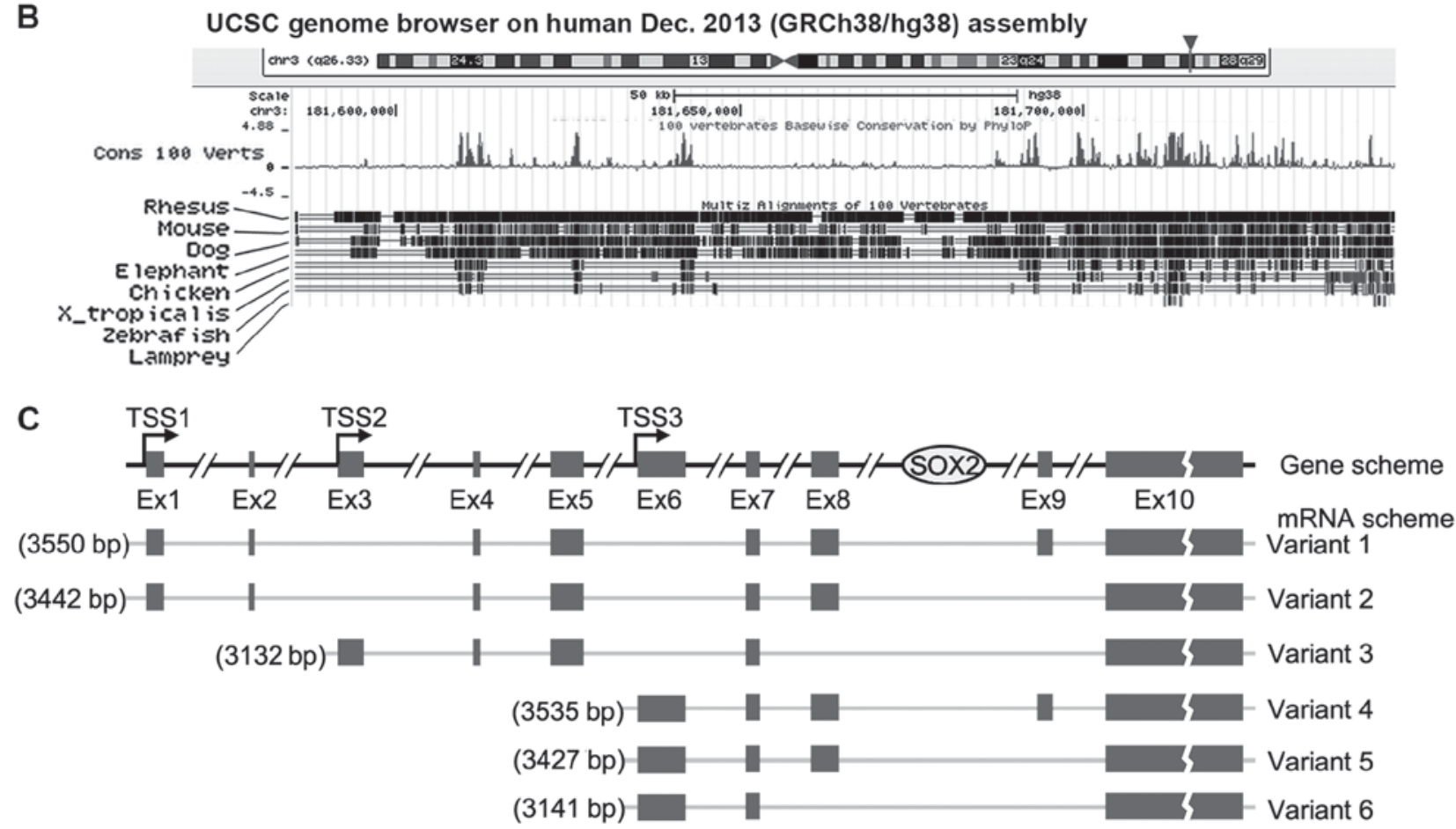

Figure 5. Genome structure and conservation analysis of SOX2OT. Genome structure of SOX2OT in mice (A) and humans (B) demonstrating the gene location and conservation sites between species. Data were downloaded from http://genome.ucsc.edu. (C) The structure diagrams of SOX2OT variants according to NCBI database. TSSs, transcription starting sites; Ex, exon; SOX2OT, SOX2 original transcript.

which may have an important function in DN. However, to the best of our knowledge, its role in DN has never been reported previously. One way of investigating the role of an lncRNA in a certain disease is to study their putative co-expressed mRNAs. All the SOX2OT-associated mRNAs were analyzed in GO enrichment (Fig. 4B). Notably, results demonstrated a very similar expression pattern to that presented in Fig. 2A. Those enriched biological processes in DN, including glutathione biosynthetic processes, glucuronic and flavonoid associated processes, chylomicron, and androgen metabolic processes were also enriched in SOX2OT-associated mRNAs. These results indicated that, by serving a central part in dysregulated biological processes, SOX2OT may have a critical role in DN.

SOX2OT is highly conserved between mice and humans. Since SOX2OT is evolutionarily conserved (22) and was the most altered lncRNA in the present study, SOX2OT was investigated in the present study. The gene structure of SOX2OT in mouse and human from UCSC is presented in Fig. 5A and B. SOX2OT is located on chromosome 3 in the two species and it overlaps with mRNA encoding gene SOX2. Conservation tracking demonstrates that SOX2OT is highly conserved between different vertebrates, especially in mice and humans. SOX2OT has a number of transcript variants. Human variants 1-6 have been documented in the NCBI database and the structure diagrams are presented in Fig. 5C. A total of three transcription starting sites (TSSs) are demonstrated and between Ex (exon) 8 and 9, which is where SOX2 resides.

Expression pattern of SOX2OT in HPCs and HMCs. Since podocytes and mesangial cells are the two main types of cells functionally associated with the onset of DN, HPCs and HMCs were cultured to check SOX2OT expression. HG (30 mM for $24 \mathrm{~h}$ ) could significantly downregulate SOX2OT expression compared with the control group $(\mathrm{P}=0.0006$ in HPC group and 0.0043 in HMC group, Fig. 6A). This suggested that SOX2OT may have the same expression pattern and function in human podocytes and HMCs as that in mice. Then FISH was performed (Fig. 6B). Results demonstrated consistently weakened signal in HG-stimulated HPCs and HMCs. 


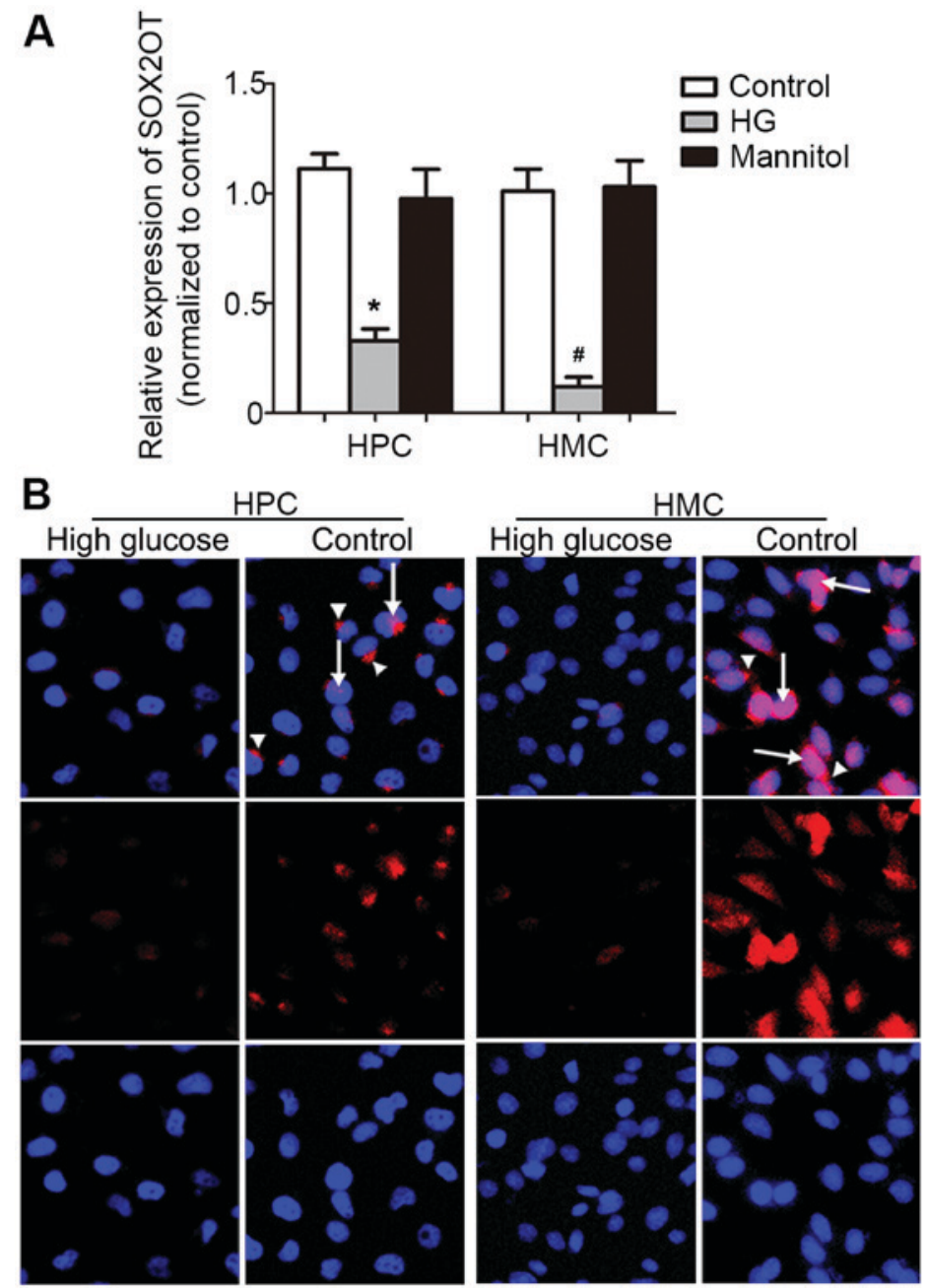

Figure 6. Expression and location of SOX2OT in HPCs and HMCs. (A) HPCs and HMCs were cultured and stimulated by HG. Expression of SOX2OT was determined by reverse transcription quantitative polymerase chain reaction. Data are presented as the mean relative expression level \pm standard deviation. ${ }^{*} \mathrm{P}=0.0006$ vs. Control HPC; ${ }^{~} \mathrm{P}=0.0043$ vs. Control HMC. (n=3). (B) Fluorescence in situ hybridization was performed in cultured HPCs and HMCs. Arrows indicate that SOX2OT was expressed in the nucleus and the arrowhead indicates that it was expressed in the cytoplasm (magnification, x200). HPCs, human podocyte cells; HMCs, human mesangial cells; HG, high glucose treatment; SOX2OT, SOX2 original transcript.

SOX2OT expressed in the nucleus (arrow) and cytoplasm (arrow head). However, in HPCs, SOX2OT was expressed in the cytoplasm, while in HMCs, it was expressed in the nucleus. This very different distribution indicated that SOX2OT may have different roles in HPCs and HMCs under HG-stimulation.

\section{Discussion}

For decades, IncRNA has always been recognized as byproduct of the gene transcription. Although recent studies (23-25) have greatly improved current knowledge about the function and associated mechanism of lncRNA, it is mostly limited to the area of oncology. The role of lncRNA in renal diseases is largely unknown.

In the present study, $\mathrm{db} / \mathrm{db}$ mice were used as a spontaneous diabetes model. Sustained high blood glucose ends in the occurrence of protein urea and finally the onset of DN. Microarray analysis using the renal cortex of $\mathrm{db} / \mathrm{db}$ mice revealed large number of dysregulated lncRNAs and mRNAs. To investigate what biological processes and pathways were most altered GO and KEGG pathway enrichment analysis was carried out. As expected, metabolic associated biological processes were altered, including glucuronic associated processes. The significant cellular component enrichment of chylomicron was consistent with previous studies that demonstrated lipid dysregulation served an important role in DN $(26,27)$. It is already well known that oxidative stress is associated with the onset and progression of DN (28). The present study's result also added novel evidence to the role of oxidative stress by describing alterations in certain known functional processes or pathways, including flavonoid-associated processes (29), cytochrome P450 (30) and glutathione metabolism (31). Alterations in other DN-associated pathways (32), including the renin-angiotensin system, PPAR signaling pathway and arachidonic acid metabolism were also demonstrated in the present study. These discoveries, on one hand, proved the successful establishment of the DN model at the molecular level and on the other hand they provided a whole picture of disordered gene expression, which indicates novel directions for future study. In addition, except for the commonly enriched pathways, steroid-related alterations were also demonstrated in GO and pathway enrichment maps. At present, steroids are widely used for treatment of diverse glomerular diseases. Although advanced stages of DN are often accompanied by 
massive proteinuria, steroid hormones are rarely used in these patients due to side effects or uncertain effectiveness. However the results of the present study, together with other microarray analysis (33) suggested that steroid hormone disorders may also participate in the onset of DN, which requires further study in the future.

As is currently known, the common way for lncRNA to function is via interacting with mRNA. In the present study, the lncRNA-associated mRNA alterations were also discussed which could help prediction of the lncRNA-associated cellular functions. In the present study, the correlation of differentially expressed lncRNAs and mRNAs were presented as networks according to calculated $\mathrm{PC}$ values.

RT-qPCR was introduced to validate microarray results and a total of 10 lncRNAs and mRNAs were included. In these lncRNAs, PVT1 and SOX2OT have already been reported and researched in humans, especially in cancer $(20,21)$. PVT1 has also been studied intensively in DN (34). However, the function of SOX2OT in DN has not been identified. Therefore, in the following studies, the role of SOX2OT was focused on in DN. GO enrichment was carried out again using co-expressed mRNAs of SOX2OT. As expected, enrichment results were highly consistent with GO and KEGG results. Flavonoid associated processes, oxidoreductase reaction, glutathione metabolism and steroid metabolic processes were all exhibited, indicating that SOX2OT is involved in the major disorders of DN. Since the role of SOX2OT in DN has not been reported previously, it is of interest to investigate the exact function, which may provide a novel point of view on DN treatment.

The location within the genome of SOX2OT is chromosome 3 in humans and mice. Conservational analysis demonstrated that SOX2OT is conserved in multiple vertebrates. This conservation among species indicated that SOX2OT may have an important role in normal physiology. SOX2OT has a number of variants (35). At least 6 versions were documented in NCBI. A total of three TSSs were demonstrated to start transcription of different variants. However, the overlapping sequences prevented the design of specific and highly efficient primers. As a result, only the total amount of SOX2OT was measured. SOX2OT was first reported to be associated with cell proliferation in breast cancer, lung cancer, colorectal cancer and gastric cancer (36-38). Su et al (39) proposed that SOX2OT acted as a microRNA (miR)-194-5p or miR-122 sponge and knockdown of SOX2OT inhibited the malignant biological behaviors of glioblastoma stem cell. Recently, it is reported that, in diabetes-induced retinal neurodegeneration, SOX2OT expression is significantly downregulated in the retinas of STZ-induced diabetic mice and in the Retinal Ganglion Cells upon HG or oxidative stress (40). Diabetic-induced nephropathy is also a micro-vascular complication and SOX2OT was also downregulated in the mice renal cortex and cultured HPCs and HMCs. FISH was performed to find out the cellular location. The images exhibited downregulation of SOX2OT under HG stimulation. Furthermore, it was demonstrated that the location of SOX2OT was very different in these two cells, suggesting that SOX2OT may serve different functions in different cells. Considering the conservation of SOX2OT between mice and humans, the consistent result of SOX2OT suggested it also plays a role in human DN. However, the role of SOX2OT in certain cell types needs further investigation.
In conclusion, the present study confirmed that $\mathrm{db} / \mathrm{db}$ mice exhibited different gene expression compared with $\mathrm{db} / \mathrm{m}$ mice and these DEGs were involved in diverse biological processes and pathways associated with DN. Out of these dysregulated lncRNAs, the significantly different and species-conserved lncRNA SOX2OT were identified to be the putative controller of DN onset and progression. SOX2OT may serve a central role in orchestrating scenarios of molecular disorder and be the putative target for DN treatment. The detailed function and role of SOX2OT in DN requires further study in the future.

\section{Acknowledgements}

Not applicable.

\section{Funding}

The present study was supported by the National Natural Science Foundation of China (grant nos. 81570690 and 81700633), the Excellent Youth Foundation of Henan Scientific Committee (grant no. 154100510017), the Youth Foundation of the First Affiliated Hospital of Zhengzhou University to Jin Shang, Key Scientific Research Projects of Henan Colleges and Universities (grant no. 17A320065), Foundation and Frontier Technology Research Program of Henan Province (grant no. 142300410211).

\section{Availability of data and materials}

The datasets used and/or analyzed during the current study are available from the corresponding author on reasonable request.

\section{Authors' contributions}

$\mathrm{ZZ}$ and JX conceived and designed the experiments; JS and XW performed the experiments; GC and DL analyzed the data; XZ and $\mathrm{YJ}$ analyzed and interpreted data and wrote the manuscript; $\mathrm{ZZ}$ revised and gave final approval of the manuscript to be published. All authors read and approved the final manuscript.

\section{Ethics approval and consent to participate}

All protocols were approved by the Institutional Animal Care and Use Committee of the First Affiliated Hospital of Zhengzhou University and conducted in accordance with the National Institutes of Health Guide for the Care and Use of Laboratory Animals.

\section{Patient consent for publication}

Not applicable.

\section{Competing interests}

The authors declare that they have no competing interests.

\section{References}

1. Vicente PC, Kim JY, Ha JJ, Song MY, Lee HK, Kim DH, Choi JS and Park KS: Identification and characterization of site-specific $\mathrm{N}$-glycosylation in the potassium channel Kv3.1b. J Cell Physiol 233: 549-558, 2018. 
2. Badal SS and Danesh FR: New insights into molecular mechanisms of diabetic kidney disease. Am J Kidney Dis 63 (2 Suppl 2): S63-S83, 2014.

3. Hwang SH, Han BI and Lee M: Knockout of ATG5 leads to malignant cell transformation and resistance to Src family kinase inhibitor PP2. J Cell Physiol 233: 506-515, 2018.

4. Frenette-Cotton R, Marcoux AA, Garneau AP, Noel M and Isenring P: Phosphoregulation of $\mathrm{K}(+)-\mathrm{Cl}(-)$ cotransporters during cell swelling: Novel insights. J Cell Physiol 233: 396-408, 2018.

5. Shang J, Wan Q, Wang X, Duan Y, Wang Z, Wei X, Zhang Y, Wang $\mathrm{H}$, Wang $\mathrm{R}$ and $\mathrm{Yi} \mathrm{F}$ : Identification of NOD2 as a nove target of RNA-binding protein HuR: Evidence from NADPH oxidase-mediated HuR signaling in diabetic nephropathy. Free Radic Biol Med 79: 217-227, 2015.

6. Liu R, Liao X, Li X, Wei H, Liang Q, Zhang Z, Yin M, Zeng X, Liang $\mathrm{Z}$ and $\mathrm{Hu} \mathrm{C}$ : Expression profiles of long noncoding RNAs and mRNAs in post-cardiac arrest rat brains. Mol Med Rep 17: 6413-6424, 2018.

7. Liao Z, Zhao J and Yang Y: Downregulation of lncRNA H19 inhibits the migration and invasion of melanoma cells by inactivating the NFkappaB and PI3K/Akt signaling pathways. Mol Med Rep 17: 7313-7318, 2018.

8. Xu JH, Chang WH, Fu HW, Yuan $\mathrm{T}$ and Chen P: The mRNA, miRNA and lncRNA networks in hepatocellular carcinoma: An integrative transcriptomic analysis from Gene Expression Omnibus. Mol Med Rep 17: 6472-6482, 2018

9. Li Z, Xu Y, Liu X, Nie Y and Zhao Z: Urinary heme oxygenase-1 as a potential biomarker for early diabetic nephropathy. Nephrology (Carlton) 22: 58-64, 2017.

10. Zhang Z, Cheng X, Yue L, Cui W, Zhou W, Gao J and Yao H: Molecular pathogenesis in chronic obstructive pulmonary disease and therapeutic potential by targeting AMP-activated protein kinase. J Cell Physiol 233: 1999-2006, 2018.

11. Guo J, Xiao J, Gao H, Jin Y, Zhao Z, Jiao W, Liu Z and Zhao Z: Cyclooxygenase-2 and vascular endothelial growth factor expressions are involved in ultrafiltration failure. J Surg Res 188: $527-536 \mathrm{e} 2,2014$

12. Tian F, Gu C, Zhao Z, Li L, Lu S and Li Z: Urinary Emmprin, matrix metalloproteinase 9 and tissue inhibitor of metalloproteinase 1 as potential biomarkers in children with ureteropelvic junction narrowing on conservative treatment. Nephrology (Carlton) 20: 194-200, 2015.

13. Kong XD, Shi HR, Liu N, Wu QH, Xu XJ, Zhao ZH, Lu N, Li-Ling $\mathrm{J}$ and Luo D: Mutation analysis and prenatal diagnosis for three families affected by isolated methylmalonic aciduria. Genet Mol Res 13: 8234-8240, 2014.

14. Zou Y, Li C, Shu F, Tian Z, Xu W, Xu H, Tian H, Shi R and Mao X: lncRNA expression signatures in periodontitis revealed by microarray: The potential role of IncRNAs in periodontitis pathogenesis. J Cell Biochem 116: 640-647, 2015.

15. Zhang S, Zhang Y, Wei X, Zhen J, Wang Z, Li M, Miao W, Ding H, Du P,Zhang W, et al: Expression and regulation of a novel identified TNFAIP8 family is associated with diabetic nephropathy. Biochim Biophys Acta 1802: 1078-1086, 2010

16. Livak KJ and Schmittgen TD: Analysis of relative gene expression data using real-time quantitative PCR and the 2(-Delta Delta C(T)) method. Methods 25: 402-408, 2001

17. Shi X, Xu Y, Zhang C, Feng L, Sun Z, Han J, Su F, Zhang Y, Li C and Li X: Subpathway-LNCE: Identify dysfunctional subpathways competitively regulated by lncRNAs through integrating lncRNA-mRNA expression profile and pathway topologies. Oncotarget 7: 69857-69870, 2016.

18. Chen X: KATZLDA: KATZ measure for the lncRNA-disease association prediction. Sci Rep 5: 16840, 2015.

19. Hu M, Wang R, Li X, Fan M, Lin J, Zhen J, Chen L and Lv Z LncRNA MALAT1 is dysregulated in diabetic nephropathy and involved in high glucose-induced podocyte injury via its interplay with $\beta$-catenin. J Cell Mol Med 21: 2732-2747, 2017.

20. Wang C, Han C, Zhang Y and Liu F: LncRNA PVT1 regulate expression of HIFlalpha via functioning as ceRNA fo miR199a5p in nonsmall cell lung cancer under hypoxia. Mol Med Rep 17: 1105-1110, 2018.

21. Tang X, Gao Y, Yu L, Lu Y, Zhou G, Cheng L, Sun K, Zhu B, $\mathrm{Xu} \mathrm{M}$ and Liu J: Correlations between IncRNA-SOX2OT polymorphism and susceptibility to breast cancer in a Chinese population. Biomark Med 11: 277-284, 2017.

22. Shahryari A, Jazi MS, Samaei NM and Mowla SJ: Long non-coding RNA SOX2OT: Expression signature, splicing patterns, and emerging roles in pluripotency and tumorigenesis. Front Genet 6: 196, 2015
23. Munschauer M, Nguyen CT, Sirokman K, Hartigan CR, Hogstrom L, Engreitz JM, Ulirsch JC, Fulco CP, Subramanian V, Chen J, et al: The NORAD lncRNA assembles a topoisomerase complex critical for genome stability. Nature 561: 132-136, 2018.

24. Zhang Y, Pitchiaya S, Cieslik M, Niknafs YS, Tien JC, Hosono Y, Iyer MK, Yazdani S, Subramaniam S, Shukla SK, et al: Analysis of the androgen receptor-regulated lncRNA landscape identifies a role for ARLNC1 in prostate cancer progression. Nat Genet 50: 814-824, 2018.

25. Hua JT, Ahmed M, Guo H, Zhang Y, Chen S, Soares F, Lu J, Zhou S, Wang M, Li H, et al: Risk SNP-mediated promoter-enhancer switching drives prostate cancer through IncRNA PCAT19. Cell 174: 564-575e18, 2018.

26. Wong R, Chen W, Zhong X, Rutka JT, Feng ZP and Sun HS Swelling-induced chloride current in glioblastoma proliferation, migration, and invasion. J Cell Physiol 233: 363-370, 2018.

27. Bagheri V, Memar B, Momtazi AA, Sahebkar A, Gholamin M and Abbaszadegan MR: Cytokine networks and their association with Helicobacter pylori infection in gastric carcinoma. J Cell Physiol 233: 2791-2803, 2018.

28. Feng B, Yan XF, Xue JL, Xu L and Wang H: The protective effects of alpha-lipoic acid on kidneys in type 2 diabetic Goto-Kakisaki rats via reducing oxidative stress. Int J Mol Sci 14: 6746-6756, 2013.

29. Olli KE, Li K, Galileo DS and Martin-DeLeon PA: Plasma membrane calcium ATPase 4 (PMCA4) co-ordinates calcium and nitric oxide signaling in regulating murine sperm functional activity. J Cell Physiol 233: 11-22, 2018.

30. Noda S, Sumita Y, Ohba S, Yamamoto H and Asahina I: Soft tissue engineering with micronized-gingival connective tissues. J Cell Physiol 233: 249-258, 2018.

31. Lin W, Izu Y, Smriti A, Kawasaki M, Pawaputanon C, Bottcher RT, Costell M, Moriyama K, Noda M and Ezura Y: Profilin1 is expressed in osteocytes and regulates cell shape and migration. J Cell Physiol 233: 259-268, 2018.

32. Xu HZ, Cheng YL, Wang WN, Wu H, Zhang YY, Zang CS and Xu ZG: 12-Lipoxygenase inhibition on microalbuminuria in Type- 1 and Type-2 diabetes is associated with changes of glomerular angiotensin II Type 1 receptor related to insulin resistance. Int J Mol Sci 17: pii: E684, 2016.

33. Chen S, Dong C, Qian X, Huang S, Feng Y, Ye X, Miao H, You Q, Lu Y and Ding D: Microarray analysis of long noncoding RNA expression patterns in diabetic nephropathy. J Diabetes Complications 31: 569-576, 2017.

34. Alvarez ML and Distefano JK: The role of non-coding RNAs in diabetic nephropathy: Potential applications as biomarkers for disease development and progression. Diabetes Res Clin Pract 99: 1-11, 2013.

35. Strippoli R, Loureiro J, Moreno V, Benedicto I, Lozano ML, Barreiro O, Pellinen T, Minguet S, Foronda M, Osteso MT, et al: Caveolin-1 deficiency induces a MEK-ERK1/2-Snail-1-dependent epithelial-mesenchymal transition and fibrosis during peritoneal dialysis. EMBO Mol Med 7: 357, 2015.

36. Askarian-Amiri ME, Seyfoddin V, Smart CE, Wang J, Kim JE, Hansji H, Baguley BC, Finlay GJ and Leung EY: Emerging role of long non-coding RNA SOX2OT in SOX2 regulation in breast cancer. PLoS One 9: e102140, 2014.

37. Hou Z, Zhao W, Zhou J, Shen L, Zhan P, Xu C, Chang C, Bi H, Zou J, Yao X, et al: A long noncoding RNA Sox 2ot regulates lung cancer cell proliferation and is a prognostic indicator of poor survival. Int J Biochem Cell Biol 53: 380-388, 2014.

38. Liu S, Xu B and Yan D: Enhanced expression of long non-coding RNA Sox 2ot promoted cell proliferation and motility in colorectal cancer. Minerva Med 107: 279-286, 2016.

39. Su R, Cao S, Ma J, Liu Y, Liu X, Zheng J, Chen J, Liu L, Cai H, et al: Knockdown of SOX2OT inhibits the malignant biological behaviors of glioblastoma stem cells via up-regulating the expression of miR-194-5p and miR-122. Molecular cancer 16: $171,2017$.

40. Li CP, Wang SH, Wang WQ, Song SG and Liu XM: Long Noncoding RNA-Sox2OT knockdown alleviates diabetes mellitus-induced retinal ganglion cell (RGC) injury. Cell Mol Neurobiol 37: 361-369, 2017.

This work is licensed under a Creative Commons Attribution 4.0 International (CC BY-NC 4.0) License 